\title{
POSICIONAMENTO POR PONTO PRECISO E POSICIONAMENTO RELATIVO COM GNSS: QUAL É O MÉTODO MAIS ACURADO ATUALMENTE?
}

\section{Precise point positioning and relative positioning with GNSS: what most accurate currently?}

\author{
Marília Sanglard Almeida ${ }^{1}$ \\ William Rodrigo Dal Poz 1 \\ 1 Universidade Federal de Viçosa - UFV, Centro de Ciências Exatas e Tecnológicas - Departamento de \\ Engenharia Civil, Campus Universitário - CEP:36.570-000 - Viçosa/MG - \\ Brasil.Email:marilia.almeida@ufv.br;dalpoz@gmail.com.
}

\section{Resumo:}

O posicionamento relativo ainda é o método de posicionamento mais utilizado para determinação de coordenadas em levantamentos geodésicos de precisão. Contudo, o Posicionamento por Ponto Preciso (PPP) está cada vez mais em evidência, em função dos bons resultados oferecidos e pela praticidade de seu uso. Este trabalho tem como objetivo avaliar a acurácia destes métodos de levantamento, com o intuito de indicar qual método de posicionamento com GNSS é mais acurado atualmente. Os dados utilizados neste trabalho foram coletados pelas estações da RBMC (Rede Brasileira de Monitoramento Contínuo dos Sistemas GNSS). Para análise do PPP foi utilizado o serviço gratuito online IBGE-PPP, e para análise do posicionamento relativo estático foram utilizados o serviço de posicionamento online gratuito AUSPOS e o software comercial LGO (Leica Geo Office Combined). Após a compatibilização entre os sistemas de referência e época das coordenadas estimadas pelo LGO, IBGE-PPP e AUSPOS, estas coordenadas foram comparadas com as coordenadas de referência disponibilizadas pelo IBGE, que também foram compatibilizadas para o mesmo sistema e época de referência das coordenadas estimadas. De acordo com os resultados obtidos, conclui-se que, atualmente, o método de posicionamento relativo continua proporcionando os resultados mais acurados, independentemente do comprimento da linha de base. É importante destacar a potencialidade do uso de receptores de uma frequência no posicionamento relativo para linhas de base curtas (até $20 \mathrm{~km}$ ). Neste caso, em 64,3\% dos resultados, de um total de 147, a acurácia foi milimétrica. Deve salientar também a potencialidade do IBGE-PPP e do AUSPOS. No caso do IBGE-PPP em $100 \%$ dos casos a acurácia foi centimétrica, enquanto que no AUSPOS, em apenas $15,6 \%$ dos casos a acurácia foi decimétrica.

Palavras-chave: Acurácia, PPP, AUSPOS, Posicionamento Relativo, GNSS.

\begin{abstract}
:
Relative positioning is still the most widely used method for determination of coordinates in precision geodetic surveys. However, the Precise Point Positioning (PPP) is increasingly in evidence, due to the good results offered and the practicality of its use. This study aims to evaluate the accuracy of these positioning methods in order to indicate which method is currently more accurate. The data used in this study were collected by the stations of RBMC (Brazilian Network for Continuous Monitoring of GNSS Systems). For PPP analysis, it was used the free
\end{abstract}


service online IBGE-PPP, and for analysis of static relative positioning, it was used the free online GPS processing service AUSPOS and commercial software LGO (Leica Geo Office Combined). After the compatibility between the systems of reference and timing of coordinates estimated by LGO, IBGE-PPP and AUSPOS, these coordinates were compared with the reference coordinates provided by the IBGE, which were also transformed and updated to the same reference system and time of coordinates estimated. According to the results, it was concluded that, at present, the method of relative positioning continues to provide the most accurate results, regardless of the length of the baseline. It is important to emphasize the potentiality of the use of single frequency receivers in the relative positioning for short baselines (until $20 \mathrm{~km}$ ). In this case, at $64.3 \%$ of the results, a total of 147, the accuracy was millimeter. It should be noted the potential of the IBGE-PPP and AUSPOS. In the case of IBGE-PPP in 100\% of cases the accuracy was centimeter, while in AUSPOS in only $15.6 \%$ of cases the accuracy was decimeter.

Key Words: Accuracy, PPP, AUSPOS, Relative Positioning, GNSS.

\section{Introdução}

Tradicionalmente o posicionamento relativo tem sido empregado em levantamentos de alta precisão, alcançando níveis milimétricos. Contudo, recentemente, têm-se disponíveis os denominados serviços de PPP online, que vem ganhando destaque na geodesia, em razão da praticidade e bons resultados oferecidos. Vale lembrar que atualmente tem-se disponível vários tipos desses serviços, onde se destacam: CSRS-PPP, disponibilizado pelo NRCan (Natural Resources Canada); GAPS (GPS Analysis and Positioning Software), da UNB (University of New Brunswick); APPS (Automatic Precise Positioning Service) do JPL (Jet Propulsion Laboratory); magicGNSS da GMV Aerospace and Defense, e do próprio IBGE-PPP, que faz uso do aplicativo de processamento CSRS-PPP. Empresas fabricantes de receptores GNSS também tem desenvolvido serviços de PPP online gratuitos, como o CenterPoint da Trimble.

O posicionamento relativo, que tradicionalmente tem sido aplicado com o uso de aplicativos comerciais, também pode ser empregado com o uso de sistemas de processamento online. Dentre estes, pode-se citar como exemplo o serviço gratuito AUSPOS (AUSPOS-Online GPS Processing), o qual processa dados GPS através do posicionamento relativo estático em rede, com o uso do software científico de processamento de dados Bernese (AUSPOS, 2014).

King, Edwards \& Clarke, (2002) questionaram qual dos dois métodos era o mais apropriado para realizar determinadas tarefas utilizando dados GPS, comparando-os através de suas vantagens e desvantagens. Já se notava, então, que o PPP começava a "competir" com o posicionamento relativo. Em 2008, Ebner \& Featherstone, (2008) discutiram as desvantagens do posicionamento relativo perante o PPP, no que tange ao valor monetário para o estabelecimento e manutenção de redes de controle geodésicas. Além disso, destacou como desvantagem o fato de várias estações serem necessariamente ocupadas simultaneamente no posicionamento relativo, o que não é necessário no PPP.

Huber et al., (2010) avaliaram o PPP quanto as suas limitações e oportunidades oferecidas. Um breve histórico do desenvolvimento do PPP e a análise de seus avanços nas duas últimas décadas, com ênfase em seus potenciais e limitações atuais e possível futura direção do PPP 
foram objeto de estudo de Grinter \& Roberts (2011), concluindo que os avanços em pesquisas envolvendo o PPP tendem a oferecer uma gama de produtos cada vez melhores, principalmente no que tange à sua precisão.

Rizos et al., (2012a) fazem a seguinte indagação: PPP: está chegando ao fim a era do posicionamento relativo? Em 2013, Ocalan et al., (2013) comparou tais métodos através de serviços de posicionamento online, mostrando que suas precisões foram bastante semelhantes para os processamentos realizados. Há ainda, dentre tantos, outros estudos sobre o assunto como Shen \& Gao, (2002), King et al, (2002), Dawson et al., (2004), Wübbena et al., (2005), Ghoddousi-Fard \& Dare, (2006), Leandro et al., (2008), Alves et al., (2011), Costa et al., (2012), Rizos et al., (2012b), Cleaver, (2013) e Janssen \& Mcelroy, (2013). Nesta direção, algumas indagações podem ser feitas atualmente: Será que o PPP é mais acurado do que o posicionamento relativo? $\mathrm{O}$ posicionamento relativo continua sendo o método de posicionamento mais acurado atualmente?

Este trabalho tem como objetivo avaliar a acurácia posicional do PPP e do posicionamento relativo estático, com o objetivo de indicar qual o método de posicionamento é mais acurado atualmente. Para tanto, na aplicação da técnica PPP, foi utilizado o serviço gratuito online IBGEPPP, quanto ao posicionamento relativo, os dados GNSS foram processados de duas maneiras: utilizando o software comercial LGO (Leica Geo Office Combined) e o serviço online gratuito AUSPOS, que processa dados GPS em rede utilizando o software científico Bernese.

\section{Metodologia}

Todos os serviços online de processamento dos dados GNSS são possíveis de serem realizados graças a disponibilização dos produtos de melhor precisão do IGS (International GNSS Service), que consistem, basicamente, das efemérides precisas e dos erros dos relógios da constelação GNSS (GPS e GLONASS).

Vale ressaltar que desde o dia 07/10/2012 (semana GPS 1709) até o presente, o sistema de referência vinculado as efemérides precisas é denominado IGb08, o qual é alinhado ao ITRF2008 (Rebishchung, 2012). Desta forma, as coordenadas estimadas pelo IBGE-PPP e AUSPOS estarão referenciadas ao IGb08, na época de coleta dos dados.

No caso do processamento no LGO (posicionamento relativo), as coordenadas das estações base, disponibilizadas em SIRGAS2000, época 2000,4, devem ser transformadas e atualizadas para o IGb08, na época de coleta dos dados. Assim, as coordenadas estimadas no LGO (das estações rover) também estarão referenciadas ao mesmo sistema de referência e época das coordenadas estimadas pelo IBGE-PPP e AUSPOS.

$\mathrm{Na}$ sequência, para realizar a análise da qualidade posicional do PPP e do método relativo, as coordenadas estimadas pelos serviços utilizados foram comparadas com as coordenadas de referência das estações da RBMC (Rede Brasileira de Monitoramento Contínuo dos Sistemas GNSS), disponibilizadas pelo IBGE. Entretanto, para realizar esta análise, as coordenadas de referência da RBMC (das estações rover) também devem ser transformadas e atualizadas para o mesmo sistema de referência e época das coordenadas estimadas. Desta forma, toda a análise foi realizada em IGb08, na época de coleta dos dados. Caso esse processo de compatibilização não fosse realizado, as coordenadas estimadas pelo LGO estariam sobre a influência do 
deslocamento das placas tectônicas ao longo do tempo, desde 2000,4 até a época de coleta dos dados (2014,0 - 01/01/2014).

O processo de transformação e atualização de coordenadas utilizado neste trabalho será descrito no próximo tópico. Mais detalhes sobre o assunto podem ser encontrados em Monico (2008) e Sapucci \& Monico (2000).

\subsection{Transformação e atualização de coordenadas}

O processo de transformação e atualização de coordenadas das estações base e rover (posicionamento relativo), deve ser realizado com o objetivo de considerar o efeito de deslocamento de placa tectônica ao longo do tempo. Em outras palavras, na primeira fase deste trabalho deve-se efetuar a compatibilização entre o sistema e época de referências.

$O$ processo de transformação e atualização de coordenadas consistiu, basicamente, na compatibilização entre o SIRGAS2000 e o ITRF2000, e o IGb08 e o ITRF2008. É importante destacar que o SIRGAS2000 como um sistema de referência é idêntico a um ITRS (International Terrestrial Reference System). Além disso, o SIRGAS2000 é uma densificação regional do ITRF2000 (SIRGAS, 2015). Como já mencionado, o IGb08 é alinhado ao ITRF2008, pois eles compartilham a mesma origem, escala e orientação (Bruyninx et al., 2010; Rebischung et al., 2012). Desta forma, neste trabalho, o processo de transformação e atualização de coordenadas envolve os sistemas de referência ITRF2000 e o ITRF2008. Para isso, foram utilizados os parâmetros de transformação, listados nas Tabelas 1 e 2 .

Tabela 1: Parâmetros de transformação de ITRF2008 para ITRF2005, época 2005,0. Fonte: adaptado de (Altamimi et al., 2012).

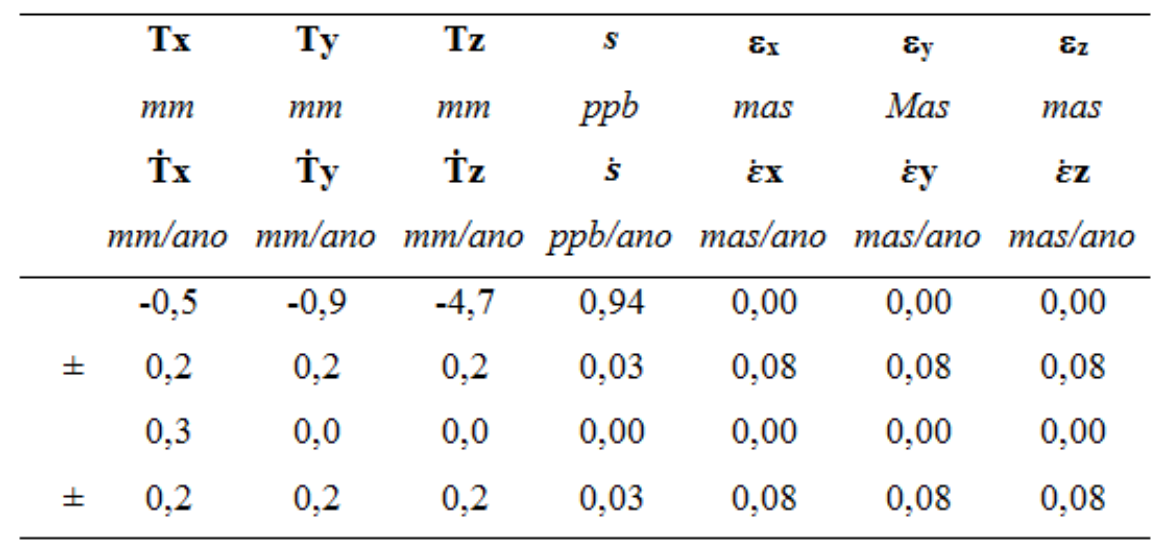


Tabela 2: Parâmetros de transformação de ITRF2005 para ITRF2000, época 2000,0. Fonte: adaptado de (Altamimi et al., 2007).

\begin{tabular}{|c|c|c|c|c|c|c|c|}
\hline & $\begin{array}{r}\mathbf{T x} \\
\mathrm{mm}\end{array}$ & $\begin{array}{c}\mathbf{T y} \\
m m\end{array}$ & $\begin{array}{r}\mathbf{T z} \\
m m\end{array}$ & $\begin{array}{c}s \\
p p b\end{array}$ & $\begin{array}{c}\boldsymbol{\varepsilon}_{\mathbf{x}} \\
\text { mas }\end{array}$ & $\begin{array}{c}\boldsymbol{\varepsilon}_{\mathbf{y}} \\
\text { Mas }\end{array}$ & $\begin{array}{c}\boldsymbol{\varepsilon}_{\mathbf{z}} \\
\text { mas }\end{array}$ \\
\hline & $\begin{array}{c}\mathbf{T} \mathbf{x} \\
\mathrm{mm} / \mathrm{ano}\end{array}$ & $\begin{array}{c}\mathbf{T} \mathbf{y} \\
\mathrm{mm} / \mathrm{ano}\end{array}$ & $\begin{array}{c}\dot{\mathbf{T} z} \\
\mathrm{~mm} / \mathrm{ano}\end{array}$ & $\begin{array}{c}\dot{\boldsymbol{s}} \\
\text { ppb/ano }\end{array}$ & $\begin{array}{c}\dot{\boldsymbol{\varepsilon}} \mathbf{x} \\
\mathrm{mas} / a n o\end{array}$ & $\begin{array}{c}\dot{\boldsymbol{\varepsilon}} \mathbf{y} \\
\mathrm{mas} / \mathrm{ano}\end{array}$ & $\begin{array}{c}\dot{\boldsymbol{\varepsilon}} \mathbf{z} \\
\mathrm{mas} / a n o\end{array}$ \\
\hline \multirow{3}{*}{ \pm} & $-0,1$ & $-0,8$ & $-5,8$ & 0,40 & 0,00 & 0,00 & 0,00 \\
\hline & 0,3 & 0,3 & 0,3 & 0,05 & 0,01 & 0,01 & 0,01 \\
\hline & $-0,2$ & 0,1 & $-1,8$ & 0,08 & 0,00 & 0,00 & 0,00 \\
\hline \pm & 0,3 & 0,3 & 0,3 & 0,05 & 0,01 & 0,01 & 0,01 \\
\hline
\end{tabular}

Onde,

- $\vec{T}$ é o vetor das translações em $\mathrm{X}, \mathrm{Y}$ e $\mathrm{Z}$ entre os dois referenciais, dadas em metros;

- $\varepsilon$ é a matriz das rotações diferenciais em torno dos eixos $\mathrm{X}, \mathrm{Y}$ e $\mathrm{Z}$, dadas em radianos;

- $s$ é o fator de escala entre os dois referenciais, adimensional, dado em ppm;

- $\dot{T} \dot{\varepsilon}$ e $\dot{s}$ são as taxas de variações em translação, rotação e fator de escala no ITRF com relação ao tempo.

A forma utilizada neste trabalho para realizar a transformação e atualização de coordenadas consiste em atualizar os parâmetros de transformação para a época de interesse, para em seguida, realizar a transformação de coordenadas, e, por fim, atualizar as coordenadas. Destaca-se que, primeiramente, o processo de transformação e atualização de coordenadas foi realizado entre os ITRF2000 (sendo desconsiderada a diferença entre ITRF2000 e SIRGAS2000) e o ITRF2005, nas épocas 2000,0 para 2000,4, e, posteriormente, foram relacionados os ITRF2005 e o ITRF2008 (sendo desconsiderada a diferença entre ITRF2008 e IGb08), nas épocas 2005,0 para 2000,4 . Por fim, foi realizada a atualização para a época de rastreio $(2000,4$ para 2014,0$)$. Neste caso, os sinais apresentados nas Tabelas 1 e 2 devem ser invertidos.

Para atualizar os parâmetros da época inicial to para a época $t$, utiliza-se a seguinte expressão (ITRF, 2015) e (Monico, 2008);

$$
P(t)=P_{t 0}+\dot{P}_{(t-t 0)} \Rightarrow\left(\begin{array}{c}
T_{X}(t) \\
T_{Y}(t) \\
T_{Z}(t) \\
S(t) \\
e_{X}(t) \\
e_{Y}(t) \\
e_{Z}(t)
\end{array}\right)=\left(\begin{array}{c}
T_{X}\left(t_{0}\right) \\
T_{Y}\left(t_{0}\right) \\
T_{Z}\left(t_{0}\right) \\
S\left(t_{0}\right) \\
\varepsilon_{X}\left(t_{0}\right) \\
\varepsilon_{Y}\left(t_{0}\right) \\
\varepsilon_{Z}\left(t_{0}\right)
\end{array}\right)+\left(\begin{array}{c}
\dot{T}_{X} \\
\dot{T}_{Y} \\
\dot{T}_{Z} \\
\dot{S} \\
\dot{e}_{X} \\
\dot{e}_{Y} \\
\dot{e}_{Z}
\end{array}\right)(t-t 0)
$$

Onde ${ }^{\dot{P}}$ é a taxa de variação dos parâmetros (ver Tabelas 1 e 2). Após a atualização dos parâmetros, realiza-se a transformação entre os sistemas de referência, através da transformação de similaridade, por meio de três translações, três rotações e um fator de escala. $\mathrm{O}$ modelo matemático para transformar as coordenadas no sistema origem (o) para o sistema destino (d) é dado por (Petit \& Luzum, 2010):

$$
\left[\begin{array}{l}
X \\
Y \\
Z
\end{array}\right]_{d}=\left[\begin{array}{l}
X \\
Y \\
Z
\end{array}\right]_{0}+\left[\begin{array}{l}
T_{X} \\
T_{Y} \\
T_{Z}
\end{array}\right]+\left[\begin{array}{lll}
S & -\varepsilon_{Z} & \varepsilon_{Y} \\
\varepsilon_{Z} & S & -\varepsilon_{X} \\
-\varepsilon_{Y} & \varepsilon_{X} & S
\end{array}\right]\left[\begin{array}{l}
X \\
Y \\
Z
\end{array}\right]_{0}
$$


É importante salientar que os sete parâmetros de transformação e as coordenadas dos pontos nos dois sistemas devem estar na mesma época.

$A$ atualização das coordenadas das estações da época $\left(\mathrm{t}_{0}\right)$ para a época de interesse $(\mathrm{t})$ pode ser realizada de acordo com as Equações 3, 4 e 5 (SIRGAS, 2014b):

$$
\begin{aligned}
& X_{t}=X_{\left(t_{n}\right)}+V_{X}\left(t-t_{0}\right) \\
& Y_{t}=Y_{\left(t_{n}\right)}+V_{Y}\left(t-t_{0}\right) \\
& Z_{t}=Z_{\left(t_{n}\right)}+V_{Z}\left(t-t_{0}\right)
\end{aligned}
$$

Onde:

t: época de interesse para a determinação das coordenadas;

$\mathrm{t}_{0}$ : época de origem das coordenadas cartesianas geocêntricas;

$\mathrm{X}(\mathrm{t}), \mathrm{Y}(\mathrm{t}), \mathrm{Z}(\mathrm{t})$ : coordenadas cartesianas geocêntricas de uma estação na época de destino;

$\mathrm{X}\left(\mathrm{t}_{0}\right), \mathrm{Y}\left(\mathrm{t}_{0}\right), \mathrm{Z}\left(\mathrm{t}_{0}\right)$ :coordenadas cartesianas geocêntricas de uma estação na época de origem.

O modelo de velocidades utilizado neste trabalho foi o recomendado pelo IERS2010, ou seja, o NNR-NUVEL-1A (No Net Rotation - Northern University Model 1A) (Xin-Hui et al., 2013). Para obtenção dos vetores de velocidade (NNR-NUVEL-1A) foi utilizado o programa online disponibilizado pela UNAVCO no seguinte endereço eletrônico: http://www.unavco.org/community_science/science-support/crustal_motion/dxdt/model.html. A Figura 1 ilustra o fluxograma utilizado para a transformação e atualização de coordenadas.

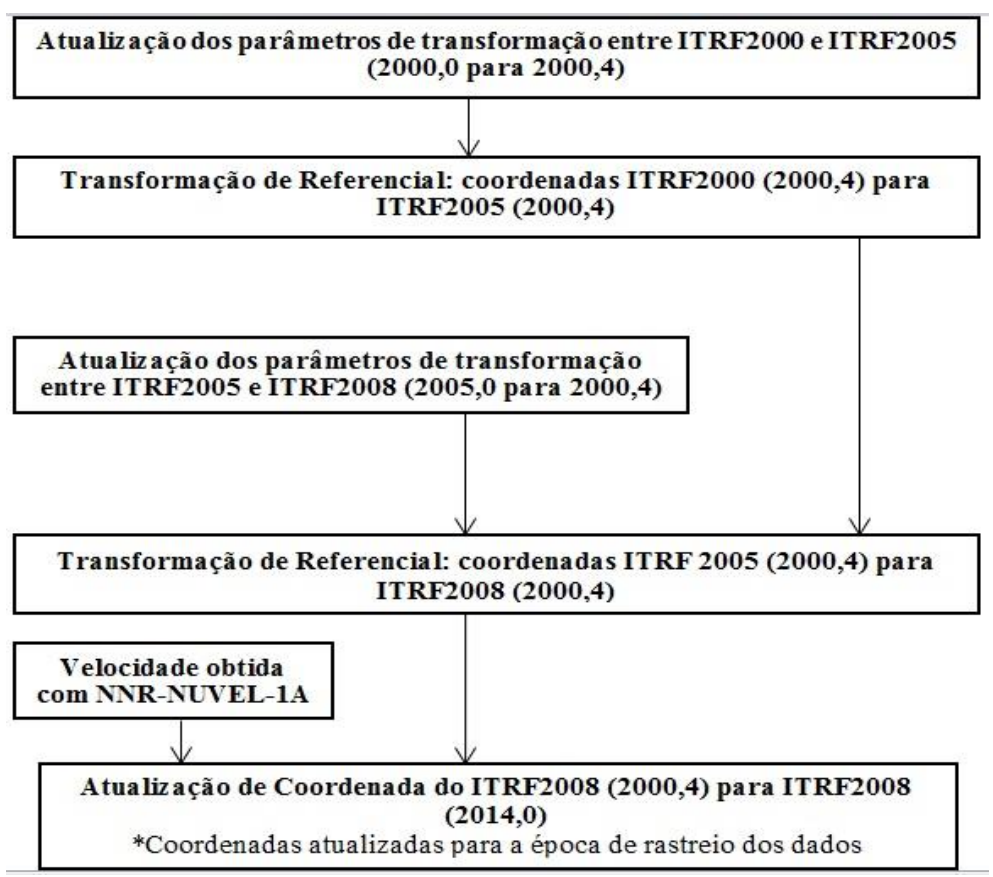

Figura 1: Esquema realizado para transformação e atualização das coordenadas utilizadas.

Essa metodologia (ITRF2000 - ITRF2005 - ITRF2008) foi sugerida por Altamimi (2012), por apresentar resultados mais acurados se comparados a transformação e atualização diretamente de ITRF2000 para ITRF2008, utilizando os parâmetros de transformação disponíveis no sítio do ITRF: http://itrf.ensg.ign.fr/doc_ITRF/Transfo-ITRF2008_ITRFs.txt. 
Para realizar a transformação entre sistemas de referência e a atualização das coordenadas de referência, foi utilizado o software Matlab, versão R2012b. Salienta-se que não foi realizada a mudança de referencial das velocidades, visto que os resultados praticamente são os mesmos. Detalhes sobre a mudança de referencial de velocidades podem ser encontrados em Monico (2008).

\subsection{Processamento de dados GNSS}

A segunda fase do trabalho envolve o processamento de dados GNSS. Os arquivos RINEX utilizados foram editados no software TEQC, onde, para cada estação, foram gerados 7 arquivos, com diferentes intervalos de rastreio $(12 \mathrm{~h}, 10 \mathrm{~h}, 8 \mathrm{~h}, 6 \mathrm{~h}, 4 \mathrm{~h}, 2 \mathrm{~h}$ e $1 \mathrm{~h}) . \mathrm{O}$ menor intervalo utilizado, correspondente a $1 \mathrm{~h}$, foi utilizado em função do AUSPOS não processar dados GPS com intervalo inferior a $1 \mathrm{~h}$. Todos os arquivos começam às 11 TUC. Em todos os processamentos foi utilizado intervalo de gravação de 30 segundos e máscara de elevação de 10 graus (editados no TEQC) e efemérides precisas. Contudo, com relação às correções dos erros envolvidos no processamento dos dados, nem todas as estratégias foram iguais, pois nos programas online de processamento (PPP e AUSPOS) o usuário não interfere na estratégia de processamento, pois todo o processo é automatizado.

Todas 46 as estações RBMC utilizadas estão na Tabela 3. Destas estações (base e rover) atualizadas e transformadas para IGb08 na época de coleta de dados, foram escolhidas 21 estações rover a serem processadas no IBGE-PPP, no AUSPOS e através do LGO. As demais estações foram utilizadas como estações base no posicionamento relativo pelo LGO.

Foram realizados 147 processamentos para o IBGE-PPP (21 estações RBMC x 7 intervalos de rastreio). O mesmo caso ocorreu para o AUSPOS. Contudo, no caso do LGO, como foram processados dados considerando seis estratégias de processamento, neste caso, foram realizados 882 processamentos (147x6). Para cada estação da RBMC tem-se um total de 56 valores de coordenadas estimadas, variando o método de processamento, a quantidade e os comprimentos das linhas de base, o intervalo de tempo de rastreio e as portadoras.

Em posse dos resultados obtidos no IBGE-PPP, calculou-se a discrepância entre as coordenadas estimadas em IGb08, época 2014,0 (época de coleta de dados), que podem ser encontradas no arquivo formato SUM nos resultados fornecidos pelo IBGE-PPP, e as coordenadas cartesianas de referência (descritivos das estações rover) atualizadas e transformadas para IGb08, época de coleta de dados. Este procedimento foi realizado de acordo com a metodologia descrita no tópico 2.1 .

Para o processamento dos dados o AUSPOS emprega o software de processamento de dados cientifico Bernese, desenvolvido pela AIUB (Astronomical Institute of the University of Bern). Para o processamento do posicionamento relativo em rede o AUSPOS utiliza as estações do IGS. Detalhes sobre esse programa podem ser acessados em < http://www.bernese.unibe.ch/>. No AUSPOS as coordenadas estimadas em IGb08, na época de coleta dos dados, também foram comparadas com os valores das coordenadas de referência de forma idêntica à realizada com as coordenadas estimadas pelo IBGE-PPP.

O processamento dos dados GNSS pelo método de posicionamento relativo estático (simples linhas de base e múltiplas linhas de base) foi feito no software LGO. Para este caso, foram definidas 6 estratégias de processamento, descritas a seguir:

-Linha de base simples (single baseline) utilizando a portadora L1;

-Linha de base simples utilizando a solução ion free; 
-Duas linhas de base utilizando a portadora L1;

-Duas linhas de base utilizando a solução ion free;

-Três linhas de base utilizando a portadora L1;

-Três linhas de base utilizando a solução ion free.

Para as linhas de base simples (1LB), há 8 estações rover obtidas a partir de linhas de base com extensão de até $20 \mathrm{~km}, 5$ estações rover obtidas a partir de linhas de base com extensão entre $20 \mathrm{~km}$ e $100 \mathrm{~km}$ e 8 estações rover a partir de linhas de base acima de 100km (total de 21 estações). Apesar da dificuldade em definir o comprimento de linhas de base no posicionamento relativo, como descrito em Dal Poz \& Camargo (2008), neste trabalho, serão consideradas linhas de base curtas, com extensão de até $20 \mathrm{~km}$, linhas de base média, com extensão de até $100 \mathrm{~km}$ e longas acima de $100 \mathrm{~km}$.

As quatro últimas estratégias, com duas linhas de base (2LB) e três linhas de base (3LB), configuram-se como ajustamento de redes GPS, que envolvem múltiplas linhas de base, com o objetivo de estimar as coordenadas de apenas uma estação rover.

A Tabela 3 apresenta as estações utilizadas no processamento e o comprimento das linhas de base formadas. As combinações foram feitas da seguinte maneira:

-Processamentos com 1LB: Primeira estação-base;

-Processamentos com 2LB: Primeira e segunda estações-base;

-Processamentos com 3LB: Primeira, segunda e terceira estações-base.

Tabela 3: Estações base e rover utilizadas no processamento e os comprimentos aproximados, em quilômetros, das respectivas linhas de base formadas.

\begin{tabular}{ccc|ccc}
\hline Bases & Rover & $\begin{array}{c}\text { Comprimentos } \\
(\mathbf{k m})\end{array}$ & Bases & Rover & $\begin{array}{c}\text { Comprimentos } \\
(\mathbf{k m})\end{array}$ \\
\hline CEEU & & 0,01 & IMBT & & 71,68 \\
CEFT & BRFT & 19,16 & SCLA & SCFL & 177,3 \\
RNMO & & 190,8 & UFPR & & 248,7 \\
\hline MCLA & 2,49 & UBA1 & & 82,6 \\
GVAL & MGMC & 310,9 & POLI & SJSP & 93,8 \\
MGRP & & 366,3 & MGIN & & 109,4 \\
\hline MGUB & & 7,21 & PBCG & & 199,9 \\
MGRP & UBER & 232,6 & CRAT & PEAF & 205,3 \\
SPJA & & 260 & ALAR & & 244,5 \\
\hline SAVO & & 9,97 & SCLA & & 169,38 \\
BAI & \multirow{2}{*}{ SSA1 } & 213,6 & SCFL & IMBT & 71,68 \\
SEAJ & & 273,6 & POAL & & 314,6 \\
\hline RIOD & & 12,04 & UBA1 & & 200,4 \\
UBA1 & ONRJ & 205,2 & ONRJ & RIOD & 12,04 \\
RJCG & & 232,2 & RJCG & & 235 \\
\hline APSA & & 14,17 & MGBH & & 142,72 \\
PAAT & MAPA & 378,8 & RJCG & VICO & 194,98 \\
MABA & 636,9 & RIOD & & 232,1 \\
\hline & & & &
\end{tabular}

Bol. Ciênc. Geod., sec. Artigos, Curitiba, v. 22, nํ1, p.175 - 195, jan-mar, 2016. 
A Figura 2 ilustra como exemplo, as linhas de base formadas para processar a estação POLI. Para 1LB, processou-se MGIN-POLI; para 2LB, processou-se MGIN-POLI e SPCA-POLI; e por fim, para 3LB, processou-se MGIN-POLI, SPCA-POLI e SJSP-POLI. Em seguida, após os processamentos das simples linhas de base, efetuou-se o ajustamento da rede GPS, considerando 2LB, 3LB.

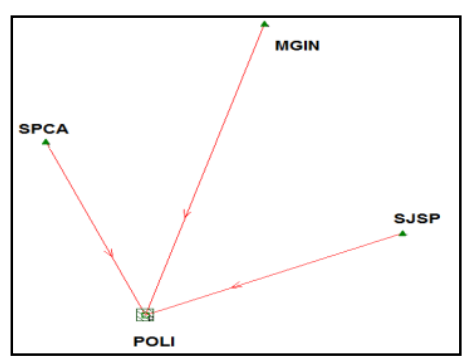

Figura 2: Linhas de base formadas para o processamento da estação POLI.

$\mathrm{O}$ arquivo de calibração absoluta das antenas das estações envolvidas foi inserido, sendo este no formato ANTEX (atx), fornecido pelo NGS (National Geodetic Survey), através do endereço: http://www.ngs.noaa.gov/ANTCAL/LoadFile?file=ngs08.atx. Este tipo de arquivo contém os valores das componentes dos deslocamentos dos centros de fase (Phase Center Offset - PCO) e das variações dos centros de fase (Phase Center Variation - PCV).

As coordenadas das estações base foram inseridas manualmente, sendo estas as coordenadas transformadas e atualizadas calculadas como descrito no tópico 2.1.

No processamento dos dados no LGO foi utilizado o modelo da troposfera de Hopfield e foram processados com as portadoras L1L2 e somente com a portadora L1. Assim, no caso da utilização das duas portadoras, foi aplicado automaticamente pelo LGO a combinação linear livre dos efeitos da ionosfera (ion free). Contudo, no caso da utilização da portadora L1, o LGO ficou configurado no modo automático, onde o principal critério para tomada de decisão se refere ao comprimento da linha de base. As demais configurações disponíveis no software ficaram no modo default.

É importante destacar que a acurácia foi calculada a partir da discrepância (tendência) em conjunto com a precisão fornecida pelos processamentos dos dados (LGO, IBGE-PPP e AUSPOS). A partir do cálculo da discrepância (calculada na época dos dados), em conjunto com a precisão fornecida (calculada na época dos dados) pelos processamentos dos dados (LGO, IBGE-PPP e AUSPOS), foi possível calcular a acurácia dos processamentos. Nota-se que todos esses cálculos foram realizados na época de coleta dos dados, com isso, o efeito de deslocamento de placas tectônicas foi considerado. Caso este procedimento não fosse realizado, os resultados obtidos pelo LGO, como mencionado anteriormente, poderiam ser precisos, porém não acurados.

\subsection{Cálculo da acurácia}

O conceito de precisão e acurácia, para Gemael (1994), Mikhail \& Ackerman (1976) e Monico et al. (2009) revela que precisão está vinculada apenas aos efeitos aleatórios ao passo que a acurácia vincula-se com os efeitos sistemáticos e aleatórios (tendência e sua dispersão). O cálculo da acurácia, proposta por Gauss, se dá por (Mikhail \& Ackerman, 1976): 


$$
m^{2}=\sigma_{p}^{2}+b^{2}
$$

Onde

$\sigma_{p}{ }^{2}=$ representa a dispersão das medidas (variância ou incerteza);

$b=$ representa a tendência ou vício do estimador (discrepância); e

$m=$ acurácia das medidas, também denominado de erro quadrático médio (mean square error).

É interessante notar que esta forma de cálculo permite avaliar a acurácia a partir de dois parâmetros independentes, tendência e precisão.

Para obtenção dos valores de acurácia das medidas, foram retirados os valores de precisão $\left(\sigma_{p}\right)$ dos relatórios de processamento, referentes a resultante planimétrica. Nos relatórios do AUSPOS e do IBGE-PPP, estes valores foram obtidos em metros ( $\left.\sigma_{\phi}, \sigma_{\lambda} e \sigma_{h}\right)$.

No caso do LGO, as coordenadas cartesianas estimadas foram convertidas para coordenadas elipsoidais. A transformação das coordenadas cartesianas em geodésicas foi realizada utilizando o método direto, proposto por Bowring (1976). Neste caso, foram adotados os parâmetros do elipsoide GRS80, com semi-eixo maior $(a)=6378137$ metros, e achatamento $(f)=$ 1/298,257222101. O GRS80 foi adotado pela Assembléia Geral da IUGG (International Union of Geodesy and Geophysics) em Canberra em 1979, e é o elipsoide recomendado atualmente.

Em seguida, com a matriz variância covariância das coordenadas cartesianas foram obtidas as precisões das coordenadas geodésicas via propagação de variância, com o uso das seguintes expressões:

$$
C_{\varnothing \lambda h(\mathrm{rad})}=D * C_{X Y Z} * D^{T}
$$

Onde D é a matriz jacobiana, dada por:

$$
D=\left[\begin{array}{ccc}
\frac{-\sin \varnothing * \cos \lambda}{(M+h)} & \frac{-\sin \phi * \sin \lambda}{(M+h)} & \frac{-\cos \phi}{(M+h)} \\
\frac{-\sin \lambda}{(M+h) * \cos \emptyset} & \frac{\cos \lambda}{(M+h) * \cos \phi} & 0 \\
\cos \phi * \cos \lambda & \cos \phi * \sin \lambda & \sin \phi
\end{array}\right]
$$

Sendo

$$
M=\frac{a *\left(1-\theta^{2}\right)}{\left(1-\varepsilon^{2} * \sin ^{2} \phi\right)^{3 / 2}}
$$

Onde,

$\mathrm{M}$ = raio de curvatura da seção meridiana;

$C_{X Y Z}=$ MVC das coordenadas cartesianas; e

$C_{\varnothing \lambda h}=$ MVC das coordenadas geodésicas.

A MVC das coordenadas geodésicas obtida forneceu os desvios-padrão da latitude e da longitude em radianos. Para transformar suas unidades para metros, basta realizar as seguintes operações matemáticas:

$$
\begin{gathered}
\sigma_{\phi(m)}=\sigma_{\phi(\mathrm{rad})} *(M+h) \\
\sigma_{\lambda(\mathrm{m})}=\sigma_{\lambda(\mathrm{rad})} *(N+h) * \cos \phi
\end{gathered}
$$

Posteriormente foram calculadas as resultantes planimétricas da precisão da seguinte forma:

$$
P=\sqrt{\sigma_{\phi(m)}^{2}+\sigma_{\lambda(m)}}
$$


Além disso, foram calculadas as discrepâncias (tendência), para posterior cálculo da acurácia. Para tanto, também foi necessária a conversão das coordenadas cartesianas em geodésicas. O cálculo da discrepância se dá por:

$$
\begin{aligned}
\Delta \phi_{\text {rad }} & =\left|\phi_{\text {calc }}-\phi_{\text {ref }}\right| \\
\Delta \lambda_{\text {rad }} & =\left|\lambda_{\text {calc }}-\lambda_{\text {ref }}\right|
\end{aligned}
$$

Onde,

$\Delta \phi_{\text {rad }}$ e $\Delta \lambda_{\text {rad }}=$ discrepâncias geodésicas calculadas na época de coleta dos dados em IGb08;

$\phi_{r e f}$ e $\lambda_{r e f}$ são as coordenadas geodésicas de referência (rover), em radianos, transformadas e atualizadas em IGb08, na época de coleta dos dados;

$\phi_{r e f}$ e $\lambda_{r e f}$ e são as coordenadas geodésicas calculadas, já estimadas em IGb08, na época de coleta dos dados;

Para a conversão das unidades para metros, pode-se fazer (Torge, 2001):

$$
\begin{gathered}
\Delta \emptyset_{(m)}=M * \Delta \phi_{\text {rad }} \\
\Delta \lambda_{(m)}=N * \Delta \lambda_{\text {rad }} * \cos \phi
\end{gathered}
$$

Em seguida, calcularam-se as resultantes planimétricas da tendência da seguinte maneira:

$$
T=\sqrt{\Delta \emptyset_{(m)}{ }^{2}+\Delta \lambda_{(m)}{ }^{2}}
$$

Onde T é a resultante planimétrica das discrepâncias (tendência), em metros. Por fim, a partir dos cálculos realizados pelas expressões 12 e 18 pode-se calcular a acurácia dada pela Equação 6.

A Figura 3 ilustra um resumo da metodologia realizada neste trabalho, desde a edição de dados no TEQC (geração de arquivos em todos os intervalos de rastreio calculados, com máscara de elevação de 10 graus e intervalo de gravação de 30 segundos) até o cálculo da acurácia. 


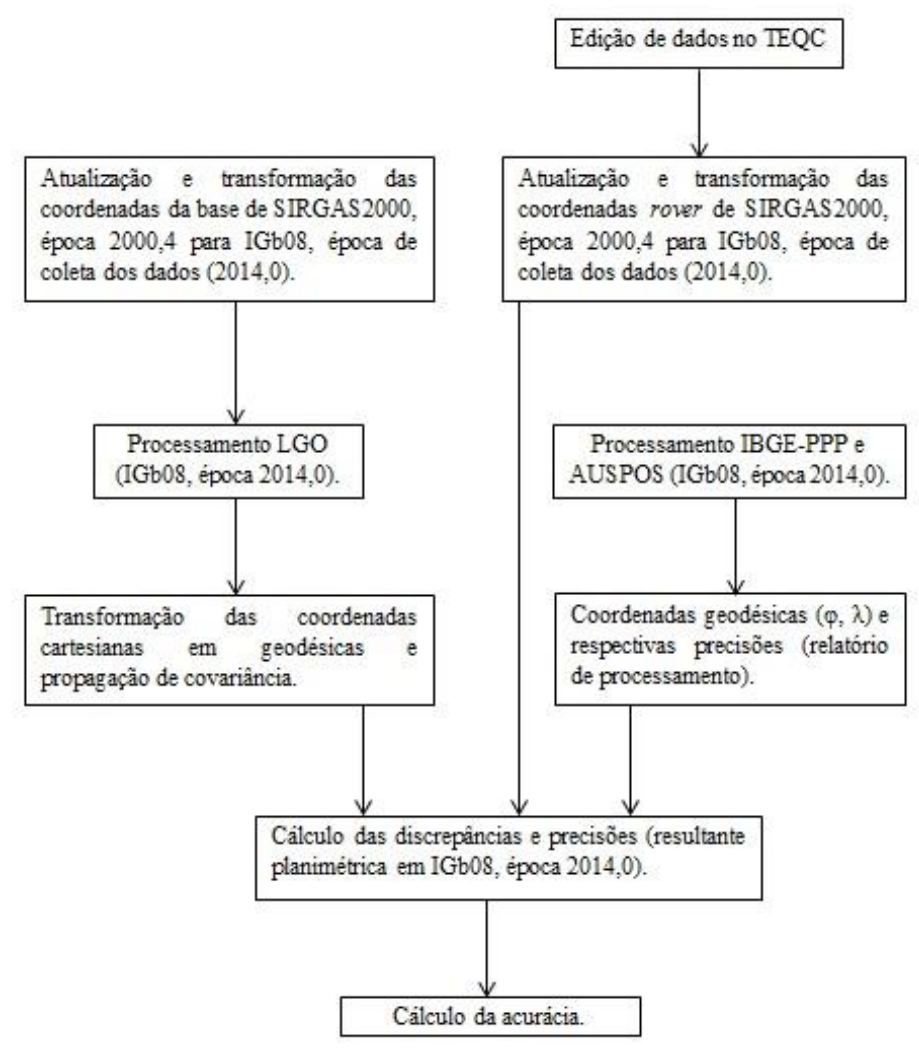

Figura 3: Fluxograma contendo a metodologia realizada no presente trabalho.

Os dados GNSS processados no IBGE-PPP e no AUSPOS foram referentes aos mesmos dados GNSS estimados no posicionamento relativo no LGO, desta forma, houve somente a necessidade de edição dos arquivos RINEX das estações rover.

\section{Resultados e análises}

Em função do limite de páginas permitido, não serão apresentados os gráficos e Tabelas de acurácia. Assim, serão mostrados os histogramas. As Figuras, numeradas de 4 a 11, apresentam os histogramas para cada tipo de processamento realizado. A Figura 4 mostra os resultados referentes ao IBGE-PPP.

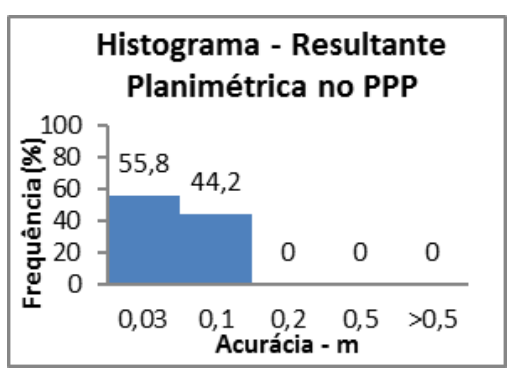

Figura 4: Histograma das resultantes planimétricas no IBGE-PPP. 
A Figura 4, que mostra o histograma de frequências da resultante planimétrica da acurácia, em metros, no PPP, são para uma amostra de 147 valores (advindos dos 147 processamentos no IBGE-PPP). Pode-se notar que $100 \%$ dos resultados são menores que $0,1 \mathrm{~m}$. Nenhum valor encontrado foi superior a $0,08 \mathrm{~m}$ (valor encontrado para a estação SSA1, com uma hora de rastreio). A Figura 5 mostra os resultados referentes ao AUSPOS.

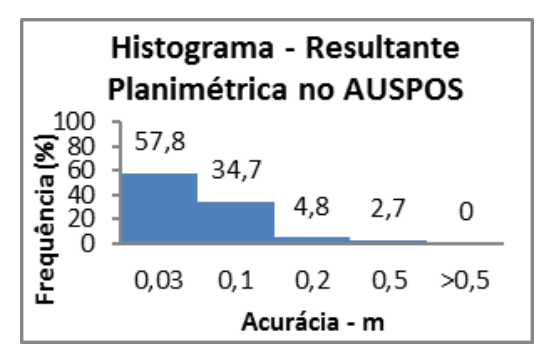

Figura 5: Histograma das resultantes planimétricas no AUSPOS.

A Figura 5, que mostra o histograma de frequências da resultante planimétrica da acurácia, em centímetros, no AUSPOS, são para uma amostra de 147 valores. Pode-se notar que 92,5\% dos resultados são menores que $0,1 \mathrm{~m}$. A partir de quatro horas de rastreio nenhum valor alcançou $0,06 \mathrm{~m}$.

A Figura 6, que mostra os histogramas de frequências da resultante planimétrica da acurácia, em metros, no LGO para 1LB, 2LB e 3LB, utilizando somente a portadora L1, são para uma amostra de 147 valores para cada caso.

Pode-se notar que, aumentando-se a quantidade de linhas de base os resultados tendem a piorar É válido lembrar que os dados utilizados para estes cálculos envolvem linhas de base curtas, médias e longas. Uma análise mais detalhada destes resultados é feita através das Figuras 7 e 8.

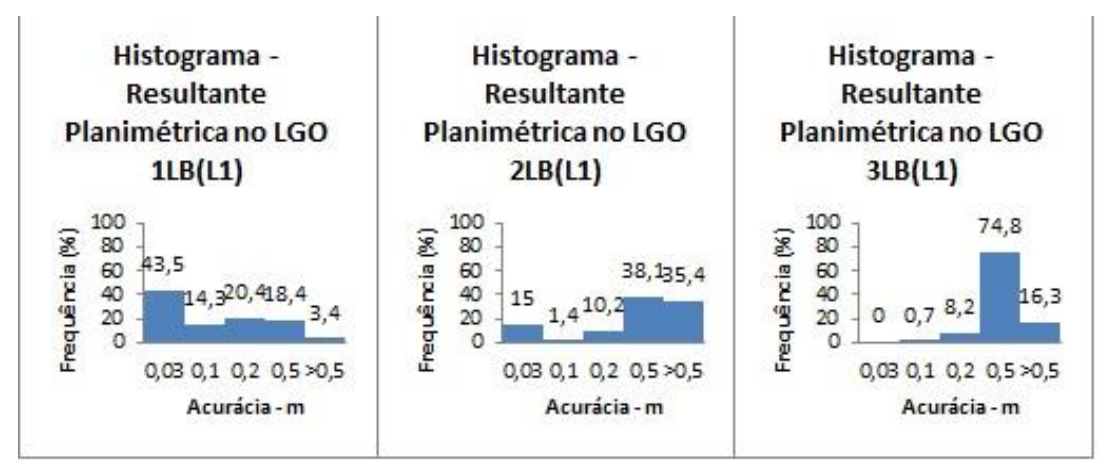

Figura 6: Histograma das resultantes planimétricas no LGO, utilizando a portadora L1, para uma, duas e três linhas de base.

A Figura 7 mostra os resultados referentes ao processamento com 1LB utilizando a portadora L1 para linhas de base curtas, em uma amostra de 56 valores. 


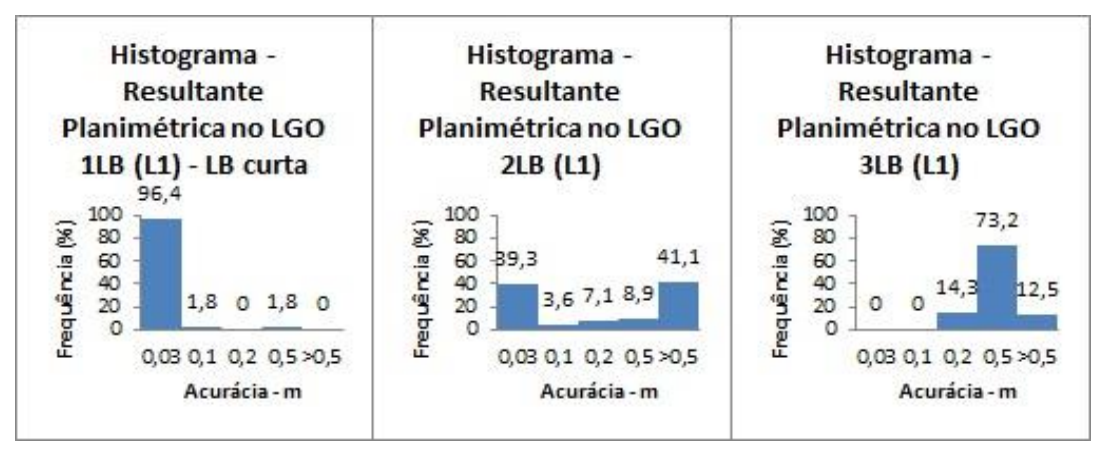

Figura 7: Histograma das resultantes planimétricas no LGO, utilizando a portadora L1, para uma (curta), duas e três linhas de base.

Analisando Figura 7, nota-se que no processamento com 1LB utilizando a portadora L1 para linhas de base curtas, 96,4\% dos resultados encontram-se abaixo de $0,03 \mathrm{~m}$. O pior resultado obtido $(0,22 \mathrm{~m})$ encontra-se para a estação SSA1 com uma hora de rastreio. A partir de duas horas de rastreio nenhum valor alcançou $0,02 \mathrm{~m}$. Isto comprova a potencialidade do uso de receptores de uma frequência em levantamento de precisão, quando são envolvidas linhas de base curtas. Vale salientar que 64,3\% dos resultados envolvendo linhas de base curtas apresentaram acurácia milimétrica.

Porém, nota-se pelos histogramas da Figura 7 que, ao acrescentar linhas de base no processamento, houve uma piora significativa nos resultados. Para os processamentos onde acrescentou-se linhas de base também curtas não houve melhora nem piora nos resultados em geral, no entanto, ao acrescentar linhas de base médias ou longas, os valores para a acurácia planimétrica aumentaram significativamente, revelando piora nos resultados.

A Figura 8 mostra os resultados referentes ao processamento com 1LB utilizando a portadora L1 para linhas de base médias e longas, em uma amostra de 91 valores.

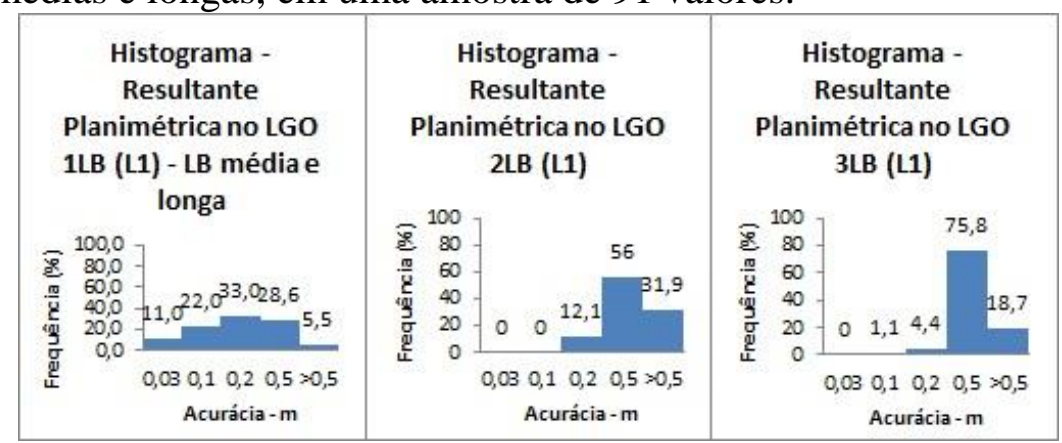

Figura 8: Histograma das resultantes planimétricas no LGO, utilizando a portadora L1, para uma (média/longa), duas e três linhas de base.

De acordo com a Figura 8, nota-se que no processamento com 1LB utilizando a portadora L1 para linhas de base médias e longas, 94,5\% dos resultados encontram-se abaixo de 0,5 m. Os piores resultados obtidos (acima de 0,2 m) encontram-se para uma, duas e quatro horas de rastreio e, a partir de seis horas de rastreio nenhum valor ultrapassou $0,24 \mathrm{~m}$.

Ao acrescentar linhas de base no processamento, percebe-se nos histogramas da Figura 8 que houve uma piora significativa nos resultados. Para os processamentos onde se acrescentou linhas de base curtas houve melhora nos resultados em geral, no entanto, ao acrescentar linhas de base médias ou longas, e após o ajustamento das redes GPS os valores para a acurácia planimétrica aumentaram, revelando piora nos resultados. 
A Figura 9 mostra os histogramas de frequências da resultante planimétrica da acurácia, em metros, no LGO para 1LB, 2LB e 3LB, utilizando a combinação ion free, para uma amostra de 147 valores, para cada caso.

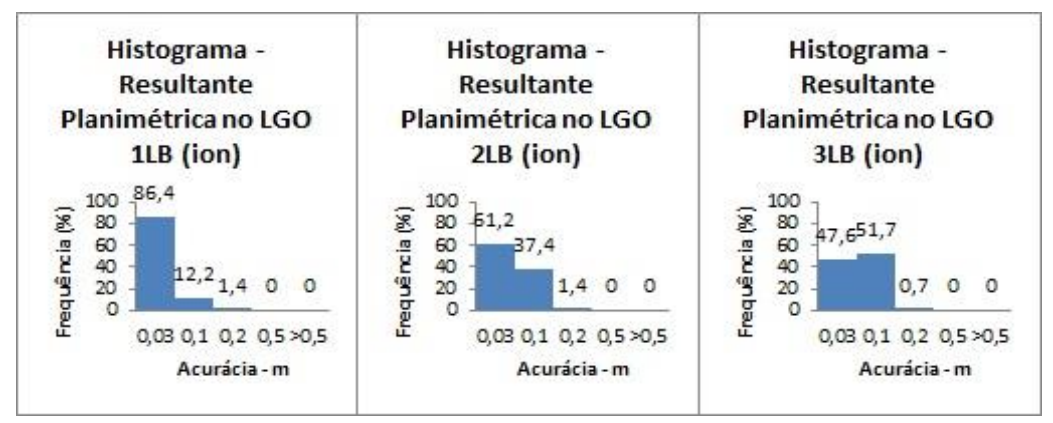

Figura 9: Histograma das resultantes planimétricas no LGO, utilizando a combinação ion free, para uma, duas e três linhas de base.

Pode-se notar que, em geral, aumentando-se a quantidade de linhas de base os resultados tendem a piorar, porém, nenhum valor ultrapassou $0,1 \mathrm{~m}$ independentemente dos comprimentos das linhas de base envolvidas, após duas horas de rastreio. É válido lembrar que os dados utilizados para estes cálculos envolvem linhas de base curtas, médias e longas. Uma análise mais detalhada destes resultados é feita através das Figuras 10 e 11.

Analisando a Figura 10, nota-se que no processamento com 1LB utilizando a combinação ion free para linhas de base curtas, $100 \%$ dos resultados encontram-se abaixo de $0,03 \mathrm{~m}$. A partir de duas horas de rastreio nenhum valor ultrapassou 0,019 m. Apenas a estação SSA1 apresentou resultados maiores que $0,02 \mathrm{~m}$ para uma hora de rastreio.

Ao acrescentar linhas de base no processamento, comparando os histogramas da Figura 10, notase uma piora nos resultados. Para os processamentos onde acrescentou-se linhas de base também curtas não houve melhora nem piora nos resultados em geral, no entanto, ao acrescentar linhas de base médias ou longas, os valores para a acurácia planimétrica aumentaram, revelando piora nos resultados.

\begin{tabular}{|c|c|c|}
\hline 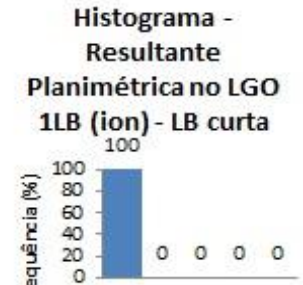 & 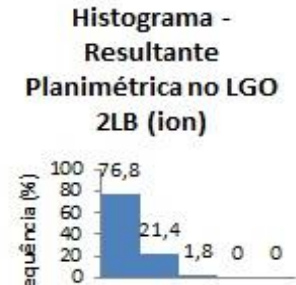 & 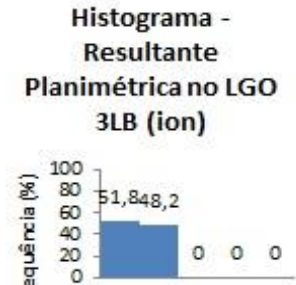 \\
\hline $\begin{array}{c}0,030,1 \quad 0,2 \quad 0,5 \times 0,5 \\
\text { Acurácia - } \mathrm{m}\end{array}$ & $\begin{array}{c}0,030,10,20,5 \times 0,5 \\
\text { Acurácia - } \mathrm{m}\end{array}$ & $\begin{array}{c}0,03 \quad 0,1 \quad 0,2 \quad 0,5>0,5 \\
\text { Acurácia- } \mathbf{m}\end{array}$ \\
\hline
\end{tabular}

Figura 10: Histograma das resultantes planimétricas no LGO, utilizando a combinação ion free, para uma (curta), duas e três linhas de base.

A Figura 11 mostra os resultados relativos ao processamento com 1LB utilizando a combinação ion free para linhas de base médias e longas. 


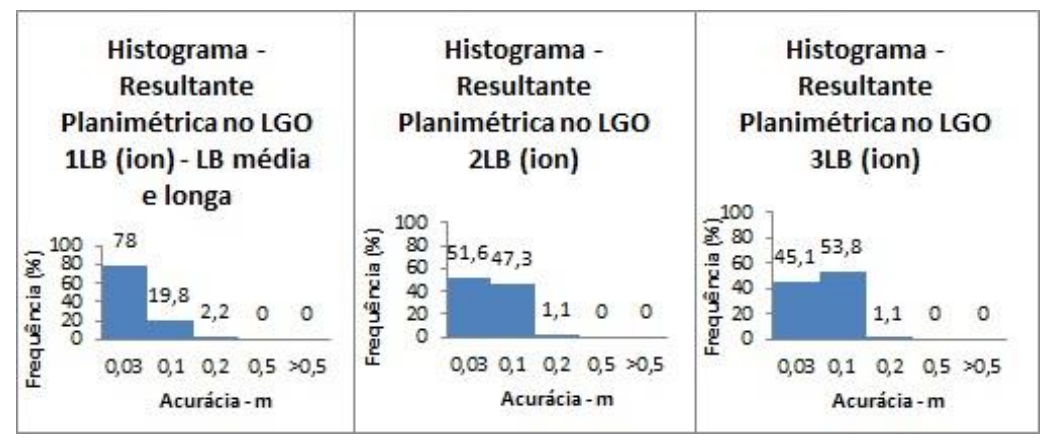

Figura 11: Histograma das resultantes planimétricas no LGO, utilizando a combinação ion free, para uma (média/longa), duas e três linhas de base.

Analisando a Figura 11, nota-se que no processamento com 1LB utilizando a combinação ion free para linhas de base médias e longas, 97,8\% dos resultados encontram-se abaixo de $0,10 \mathrm{~m}$. A partir de duas horas de rastreio nenhum valor ultrapassou $0,044 \mathrm{~m}$.

Ao acrescentar linhas de base no processamento, houve uma piora nos resultados, como ilustra a Figura 11. Para os processamentos onde acrescentou-se linhas de base também curtas não houve melhora nem piora nos resultados em geral, no entanto, ao acrescentar linhas de base médias ou longas, os valores para a acurácia planimétrica aumentaram, revelando piora nos resultados, principalmente para 3LB.

Nota-se que os resultados encontrados nas linhas de base curtas são pouco melhores que os obtidos para linhas de base médias e longas, embora a diferença entre eles seja pouco significativa (menor que $0,01 \mathrm{~m}$ na maioria dos casos).

\subsection{Vantagens e desvantagens dos métodos de posicionamento analisados}

De acordo com os histogramas apresentados, pode-se verificar que os melhores resultados obtidos são referentes ao uso do LGO, envolvendo no processamento dados de receptores de duas frequências. Pode-se verificar também que, para este caso, ocorreu a maior porcentagem de valores de acurácia menores do que apenas $0,03 \mathrm{~m}$, independentemente do comprimento da linha de base. Isso comprova que atualmente o uso de programas comerciais de processamento de dados em conjunto com o uso de receptores de dupla frequência, independentemente do comprimento da linha de base, é ainda o método mais acurado.

Contudo, algumas ponderações devem ser realizadas, apontando as vantagens e desvantagens que cada método apresenta. Diante do exposto, a Tabela 4 apresenta um resumo dessas vantagens e desvantagens. 
Tabela 4: Vantagens e desvantagens dos aplicativos computacionais utilizados neste trabalho.

\begin{tabular}{|c|c|c|}
\hline & Vantagens & Desvantagens \\
\hline AUSPOS & $\begin{array}{l}\text {-Gratuito } \\
\text {-Facilidade de uso }\end{array}$ & $\begin{array}{l}\text {-Acesso à internet } \\
\text { - Não permite alterações nas estratégias de } \\
\text { processamento } \\
\text {-Processa somente dados GPS } \\
\text {-Processa somente dados de dupla frequência } \\
\text {-Processa somente dados com mínimo de } \\
\text { uma hora de rastreio } \\
\text {-Necessidade de transformação e atualização } \\
\text { das coordenadas para o SGB }\end{array}$ \\
\hline IBGE-PPP & $\begin{array}{l}\text { - Gratuito } \\
\text { - Facilidade de uso } \\
\text {-Processa dados GLONASS e GPS } \\
\text {-Processa dados de uma ou duas } \\
\text { frequências } \\
\text {-Processa dados com qualquer } \\
\text { intervalo de rastreio } \\
\text { - Os resultados já são referenciados ao } \\
\text { SGB }\end{array}$ & $\begin{array}{l}\text {-Acesso á internet } \\
\text { •Não permite alterações nas estratégias de } \\
\text { processamento } \\
\text {-Necessidade de dados de receptores de } \\
\text { dupla frequência para posicionamento de } \\
\text { precisão }\end{array}$ \\
\hline $\begin{array}{c}\text { Leica Geo } \\
\text { Office }\end{array}$ & $\begin{array}{l}\text {-Processa dados de uma ou duas } \\
\text { frequências } \\
\text {-Processa dados com qualquer } \\
\text { intervalo de rastreio } \\
\text {-Permite alterações em suas estratégias } \\
\text { de processamento } \\
\text {-É possível realizar posicionamento } \\
\text { acurado com receptores de uma } \\
\text { frequência } \\
\text {-É possível realizar posicionamento } \\
\text { acurado com intervalo de rastreio } \\
\text { menor, em comparação com os } \\
\text { serviços de posicionamento IBGE-PPP } \\
\text { e AUSPOS. }\end{array}$ & •Não são gratuitos \\
\hline
\end{tabular}

Mesmo considerando as vantagens e desvantagens que cada método apresenta, conclui-se que o método de posicionamento relativo com o uso de aplicativo computacional comercial continua sendo a melhor opção, pois é o que apresenta o menor número de desvantagens, além de ter apresentado os melhores resultados em termos de acurácia. Embora o aplicativo não seja gratuito, o usuário pode realizar posicionamento de precisão com receptores de uma frequência, quando se envolve no processamento dos dados GNSS linhas de base curtas (até $20 \mathrm{~km}$ ). Além disso, o período de coleta de dados para posicionamento preciso é menor se comparado com os aplicativos IBGE-PPP e AUSPOS. Com isso, a médio e longo prazo o usuário pode compensar o custo do aplicativo comercial.

O IBGE-PPP se apresenta como a segunda melhor opção. Contudo, ainda há a necessidade de se utilizar receptores de duas frequências para posicionamento de precisão, com o objetivo de eliminar os efeitos de primeira ordem da ionosfera, o que não acontece quando se utiliza dados de simples frequência no PPP, sendo esta a razão de não ter sido feito nenhum experimento com esta configuração.

O aplicativo que apresentou o maior número de desvantagens foi o AUSPOS, o qualificando como a terceira melhor opção. Destaca-se que a principal desvantagem consiste na necessidade em referenciar os resultados obtidos ao SGB, algo que não ocorre com o IBGE-PPP. É importante destacar que em virtude dos dados utilizados no AUSPOS terem sido coletados no Brasil, as linhas de base formadas serão sempre muito longas, devido a carência de estações da rede IGS no Brasil. Espera-se que na Europa o AUSPOS apresente melhores resultados, pois as 
linhas de base formadas serão menores do que as formadas em território brasileiro. Dependendo da estação utilizada no processamento em território brasileiro, o comprimento da linha de base ultrapassa $5.000 \mathrm{~km}$. Mesmo nestas condições, o AUSPOS apresentou resultados muito satisfatórios.

Deve-se salientar a potencialidade do IBGE-PPP e do AUSPOS, que apresentaram bons resultados. Além disso, esses serviços de processamento são gratuitos e o usuário deve dispor de apenas um receptor. Em contrapartida, o usuário deve dispor de um receptor de dupla frequência para posicionamento de alta precisão.

\section{Conclusões e recomendações}

Conclui-se que o posicionamento relativo estático, utilizando combinação linear livre da ionosfera (uso do aplicativo computacional comercial), independente dos comprimentos e das quantidades de linhas de base envolvidas e também dos intervalos de tempo de rastreio ainda é o método de posicionamento mais acurado com GNSS, em comparação com o PPP. Devido ao fato dos processamentos utilizando a combinação ion free terem alcançado melhores resultados se comparados aos processamentos utilizando a portadora L1 envolvendo linhas de base médias e longas, fica evidenciado que o efeito ionosférico é o principal fator que afeta a acurácia do posicionamento, uma vez que com dados da portadora L1 somente não é possível eliminar os efeitos de primeira ordem da ionosfera. Além disso, à medida que se aumenta o comprimento da linha de base, outros efeitos como o da troposfera também não são totalmente eliminados, ocasionando degradação da acurácia.

É válido salientar a importância da transformação e atualização das coordenadas de referência (das bases e dos rovers), compatibilizando as épocas e sistemas de referência envolvidos, pois caso não fosse considerado o efeito do deslocamento das placas tectônicas, os valores encontrados para a discrepância posicional do LGO estariam afetados pelo deslocamento das placas, afetando, consequentemente, a acurácia posicional.

Utilizando a portadora L1, os resultados mais acurados encontram-se para linhas de base curtas, sendo estes comparáveis aos obtidos utilizando a combinação ion free, ou até melhores.

Os resultados obtidos com linhas de base simples revelaram-se melhores que os obtidos com múltiplas linhas de base, envolvendo comprimentos diferentes.

Vale salientar que o serviço online AUSPOS, embora com linhas de base envolvidas demasiadamente extensas, apresentaram valores de acurácia que estão entre os melhores resultados encontrados. Isso mostra que o serviço é eficiente independentemente dos comprimentos das linhas de base envolvidas no processamento, contudo, o AUSPOS é o serviço de posicionamento que apresentou o maior número de desvantagens.

Os serviços AUSPOS e IBGE-PPP se mostraram vantajosos no que concerne às suas precisões, além de serem gratuitos e apresentarem grande facilidade de ser utilizado pelo usuário. Em contrapartida, exigem que o mesmo tenha acesso a internet e não permitem alterações nas estratégias de processamento (máscara de elevação, latência, entre outros). Além disso, o AUSPOS só processa dados GPS, de dupla frequência, com mínimo de uma hora de rastreio.

Recomenda-se para trabalhos futuros a realização de mais experimentos envolvendo a análise de séries temporais. 


\section{AGRADECIMENTOS}

Esta pesquisa teve o incentivo da Universidade Federal de Viçosa através do Departamento de Engenharia Civil, curso de Pós-Graduação em Engenharia Civil que disponibilizou seus equipamentos, instalações e aplicativos computacionais.

$\mathrm{O}$ apoio financeiro foi dado pela CAPES através do consentimento da bolsa de estudos.

\section{REFERÊNCIAS BIBLIOGRÁFICAS}

Altamimi, Z.; Collilieux, X.; Legrand, J. Garayt, B. \& Boucher, C. ITRF2005: A new release of the International Terrestrial Reference Frame based on time series of station positions and Earth Orientation Parameters, J. Geophys. Res., 112, B09401, doi:10.1029/2007JB004949. 2007.

Altamimi, Z.; Collilieux, X. \& Métivier, L. Analysis and results of ITRF2008. (IERS Technical Note ; 37) Frankfurt am Main: Verlag des Bundesamts für Kartographie und Geodäsie, 54 pp., ISBN 978-3-86482-046-5 (print version), 2012.

Alves, C. M. D.; Monico, J. F. G.; Romão, V. M. C. Análise da acurácia no PPP a partir da solução de ambiguidades GPS em curtos períodos de ocupação. Revista Brasileira de Cartografia - RBC. № 63/5: 589-600, 2011.

AUSPOS. (2014). AUSPOS - Online GPS Processing Service (AUSPOS). Disponível em: $<$ http://www.ga.gov.au/earth-monitoring/geodesy/auspos-online-gps-processing-service.html>. Acesso em: Junho de 2014.

Bowring, B.R. Transformation from spatial to geographical coordinates. Survey Review, XXIII, 181, July, 1976.

Bruyninx, C. et al. Guidelines for EUREF densifications. Bulletin of Geodesy and Geomatics, v. 69, n. 1, p. 137-147, 2010.

Cleaver, B. L. Evaluation of the performance of web-based GNSS post processing systems. Dissertação, University of Southern Queensland. 2013.

Costa, S. M. A.; Silva, A. L.; Júnior, N. J. M. \& Lima, M. A. A. O sucesso de três anos do serviço IBGEPPP. UD y la GEOMÁTICA, n. 6, p. 79-88, 2012. Disponível em: <http://revistas.udistrital.edu.co/ojs/index.php/UDGeo/article/view/4413/6147>. Acesso em: Junho de 2014.

Dal Poz, W. R.; Camargo, P. O. Influência da variação espacial do tec na definição do comprimento de linha de base: abordagem teórica e prática. Revista Brasileira de Cartografia (RBC). ISSN 1808-0936. №60/04, dezembro. 2008.

Dawson, J; Govind, R \& Manning, J. The AUSLIG online GPS processing system (AUSPOS). The Australian surveying and land information group (AUSLIG), v. 4, 2004.

Ebner, R.; Featherstone, W. E. How well can online GPS PPP post-processing services be used to establish geodetic survey control networks? Journal of Applied Geodesy 2, 2008. Disponível em: 〈https://geodesy.curtin.edu.au/local/docs/JAG_149-157.pdf〉. Acesso em: Junho de 2014.

Gemael, C. Introdução ao ajustamento de observações: aplicações geodésicas. Curitiba. PR. Ed. UFPR, 1994. 
Ghoddousi-Fard, R \& Dare, P. Online GPS processing services: an initial study. GPS solutions, v. 10, n. 1, p. 12-20, 2006.

Grinter, T. \& Roberts, C. Precise Point Positioning: Where are we now? International Global Navigation Satellite Systems Society - IGNSS. Symposium 2011. University of New South Wales, Sydney, NSW, Australia. 15 - 17 November, 2011.

Huber, K.; Heuberger, F.; Abart, C.; Karabatic, A.; Weber R. \& Berglez, P. PPP: Precise Point Positioning - Constraints and Opportunities. FIG Congress 2010. Facing the Challenges Building the Capacity Sydney, Australia, 11-16 April, 2010.

ITRF (2015). International Terrestrial Reference Frame. Disponível em: < http://itrf.ensg.ign.fr/doc_ITRF/Transfo-ITRF2008_ITRFs.txt >. Acesso em: 05/02/2015.

Janssen, V; Mcelroy, S. Virtual RINEX: Science or fiction?. Position, n. 67, p. 38-41, 2013.

King, M.; Edwards, S.; Clarke, P. Precise Point Positioning: Breaking the Monopoly of Relative GPS Processing. Engineering Surveying Showcase - October, 2002. Disponível em: <http://www.staff.ncl.ac.uk/peter.clarke/offprints/king_etal2002.pdf>. Acesso em: Junho de 2014.

Leandro, R. F.; Langley, R. B.; Santos, M. C. GNSS data analysis in GAPS, the GPS analysis and positioning software, using IGS products. In: Proc., IGNSS Service Analysis Center Workshop, 2008.

Mikhail, E. M.; Ackermann, F. Observations and least squares. New York: A Dun-Donnelley Publisher, 1976.

Monico, J.F.G. Posicionamento pelo GNSS: Descrição, fundamentos e aplicações. São Paulo: Editora Unesp, 476 p., 2008.

Monico, J. F. G.; Póz, A. P. D.; Galo, M.; Santos, M. C.; Oliveira, L. C. Acurácia e precisão: revendo os conceitos de forma acurada. Boletim de Ciências Geodésicas, Curitiba, v. 15, p. 469483, jul. 2009.

Ocalan, T; Erdogan, B; Tunalioglu, N. Analysis of web-based online services for GPS relative and precise point positioning techniques. Boletim de Ciências Geodésicas, v. 19, n. 2, p. 191207, 2013. Disponivel em: <http://ojs.c3sl.ufpr.br/ojs2/index.php/bcg>. Acesso em: 10 jan. 2015.

Petit, G; Luzum, B. (IERS Technical Note ; 36) Frankfurt am Main: Verlag des Bundesamts für Kartographie und Geodäsie, 2010. 179 pp., ISBN 3-89888-989-6.

Rebischung, P.; Griffths, J.; Ray, J.; Schmid, R.; Collilieux, X. \& Garayt, B. IGS08: the IGS realization of ITRF2008. GPS Solutions, v. 16, n.4, 2012.

Rebischung, P. [IGSMAIL-6663] IGb08: an update on IGS08. 2012. Disponível em: http://igscb.jpl.nasa.gov/pipermail/igsmail/2012/007853.html. Acesso em: 10/01/2014.

Rizos, C.; Janssen, V.; Roberts, C. \& Grinter, T. PPP versus DGNSS. Geomatics World September / October, 2012b. Disponível em: < http://www.lpi.nsw.gov.au/_data/assets/pdf_file/0004/174937/2012_Rizos_etal_GeomaticsWor ld_206_PPP_vs_DGNSS.pdf>. Acesso em: Junho de 2014.

Rizos, C.; Janssen, V.; Roberts, C. \& Grinter, T. Precise Point Positioning: Is the Era of Differential GNSS Positioning Drawing to an End? FIG Working Week 2012 Knowing to manage the territory, protect the environment, evaluate the cultural heritage Rome, Italy, 6-10 May, 2012a. 
Sapucci, L. F.; Monico, J. F. G. Transformação de helmert generalizada no posicionamento de alta precisão: fundamentação teórica e exemplificações. Jornal Brasileiro de Geofísica, v. 18, p. 2, 2000.

Shen, X.; Gao, Y. Kinematic processing analysis of carrier phase based precise point positioning. FIG XXII International Congress. Washington, D.C., USA, April 19-26, 2002.

SIRGAS (2014b). Sistema de Referência Geocêntrico para as Américas, 2014. Disponivel em: <http://www.sirgas.org/index.php?id=15>. Acesso em: 22 jan. 2015

SIRGAS (2015) Sistema de Referência Geocêntrico para as Américas (SIRGAS). Disponível em: $<$ http://www.sirgas.org/fileadmin/docs/Boletines/Sanchez_et_al_SIRGAS_EGU2009_April_200 9_.pdf)>. Acesso em: Fevereiro de 2015.

Torge, W. Geodesy. 3rd completely ver. And extended ed. Berlim; New York: de Gruyter, 2001.

Wübbena, G.; Schmitz, M.; Bagge, A. PPP-RTK: precise point positioning using state-space representation in RTK networks. Presented at the $18^{\text {th }}$ International Technical Meeting, ION GNSS-05, Long Beach, California., September 13-16, 2005.

Xin-Hui, Z., Fu-Ping, S. And Ren, W. Establishment of the Medial-Hotspot Reference Datum and Study of the Westward Drift of Lithosphere. Chinese Journal of Geophysics, 56: 636-646. doi: 10.1002/cjg2.20059. 2013.

Recebido em março de 2015.

Aceito em maio de 2015. 